\title{
SUDDEN ONSET SENSORINEURAL HEARING LOSS: MANAGEMENT PROTOCOL
}

\author{
Anil Kumar Dash', Kabi Kanta Samantray², Rudra Narayan Biswal ${ }^{3}$
}

${ }^{1}$ Assistant Professor, Department of ENT, Kalinga Institute of Medical Sciences, KIIT Campus, Patia, Bhubaneswar. ${ }^{2}$ Associate Professor, Department of ENT, Kalinga Institute of Medical Sciences, KIIT Campus, Patia, Bhubaneswar. 3 Professor and HOD, Department of ENT, Kalinga Institute of Medical Sciences, KIIT Campus, Patia, Bhubaneswar.

\begin{tabular}{l}
\hline ABSTRACT \\
\hline AIMS \\
To study the efficacy of transcutaneous nitroglycerin and pulse steroid injection in patients with sudden hearing loss. \\
MATERIAL AND METHOD \\
This was a prospective study conducted in the Department of ENT, KIMS, Bhubaneswar; 31 patients who fit to inclusion criteria \\
included in this study.
\end{tabular}

RESULTS

Out of 31 patients of sudden onset sensorineural hearing loss, 18 (58.06\%) patients had threshold closer to $30 \mathrm{~dB}$.

\section{KEYWORDS}

Sudden Onset Sensorineural Hearing Loss, Transcutaneous Nitroglycerin Patch.

HOW TO CITE THIS ARTICLE: Dash AK, Samantray KK, Biswal RN. Sudden onset sensorineural hearing loss: management protocol. J. Evolution Med. Dent. Sci. 2016;5(45):2813-2816, DOI: 10.14260/jemds/2016/657

\section{INTRODUCTION}

Definitions of sudden hearing loss have been based on severity, time course, audiometric criteria and frequency spectrum of the loss. Abrupt as well as rapidly progressive losses have been included under a single definition of sudden hearing loss. Awakening with a hearing loss, hearing loss noted over a few days, selective low-or high-frequency loss, and distortions in speech perception have all been classified as sudden hearing losses. A commonly used criterion to qualify for this diagnosis is a sensorineural hearing loss of greater than $30 \mathrm{~dB}$ over 3 contiguous pure-tone frequencies occurring within 3 days' period. Fortunately, the vast majority of cases of sudden hearing loss are unilateral and the prognosis for some recovery of hearing is good. Usually, it presents as unilateral loss of hearing; bilateral involvement is rare and simultaneous bilateral involvement is very rare.

The postulated pathophysiology for Idiopathic Sudden Sensory Hearing Loss (ISSHL) has 4 theoretical pathways as follows:

- Labyrinthine viral infection.

- Labyrinthine vascular compromise.

- Intracochlear membrane ruptures.

- Immune-mediated inner ear disease.

A disease process involving any of these theoretical possibilities could have sudden hearing loss as a symptom. Each theory may explain a fraction of the episodes of sudden sensory hearing loss, but none of the existing theories individually could account for all episodes.

Financial or Other, Competing Interest: None.

Submission 08-04-2016, Peer Review 15-05-2016,

Acceptance 21-05-2016, Published 06-06-2016.

Corresponding Author:

Dr. Anil Kumar Dash,

Assistant Professor,

Department of ENT

Kalinga Institute of Medical Sciences,

KIIT Campus, Patia

Bhubaneswar.

E-mail: anil.dash@kims.ac.in

DOI: $10.14260 /$ jemds $/ 2016 / 657$
This is a medical emergency and various possible causal factors have been postulated. The optimal treatment modality is not known either, and this is reflected in the large number of publications suggesting the 'Best' treatment modality. Some cases will improve spontaneously. Transdermal nitroglycerin patch along with pulse steroid injections are the most recent form of treatment being studied and may provide hope for these patients, despite of the rarity and the unpredictable natural history of the disorder.

\section{MATERIAL AND METHOD}

This study was conducted in Department of Otolaryngology and HNS, KIMS, Bhubaneswar from 2012 June to June 2015; 31 patients of sudden onset hearing loss, who fit to inclusion criteria, were included in this study.

\section{Inclusion Criteria}

1. Hearing loss of greater than $30 \mathrm{~dB}$ over 3 contiguous pure-tone frequencies occurring within 3 days' period.

2. Normal tympanic membrane with type 'A' tympanometry.

3. No history of trauma to ear.

\section{Exclusion Criteria}

1. Type 'B' tympanogram.

2. Perforated tympanic membrane.

3. History of trauma to ear.

4. Cochlear otosclerosis.

After taking history and detailed otolaryngology examinations, these patients subjected to Pure Tone Audiometry and Impendence Audiometry. If the impedance is ' $A$ ' type and pure tone audiometry showing sensorineural hearing loss, then diagnosis is confirmed. All these patients were admitted to ENT ward and Intravenous Methylprednisolone $1 \mathrm{gm}$ was given for 3 days followed by oral steroid. Transdermal nitroglycerin patch, applied over forearm, started with $2.5 \mathrm{mg}$ after getting a normal ECG and cardiologist's opinion. 
The maximum dose of nitroglycerin patch is $10 \mathrm{mg}$, which is gradually increased. The maximum duration of this patch application is 10 days.

\section{RESULTS}

There were 31 patients, out of which 18 were male and 13 females in the age range from 21 to 49 with mean age of 35.35 (Standard deviation \pm 2.7 ). The impendence audiometry was performed in all 31 cases and Type ' $A$ ' type curve was seen in all these cases.

\section{The Audiometry Report as give in Table-1}

\begin{tabular}{|c|c|c|c|}
\hline $\begin{array}{c}\text { Patient } \\
\text { Serial No. }\end{array}$ & $\begin{array}{l}\text { Right Ear } \\
\text { (dB) }\end{array}$ & $\begin{array}{l}\text { Left Ear } \\
\text { (dB) }\end{array}$ & $\begin{array}{c}\text { Post Treatment } \\
\text { Improvement } \\
\text { (dB) }\end{array}$ \\
\hline 1 & 20 & 69 & 35 \\
\hline 2 & 23 & 78 & 39 \\
\hline 3 & 24 & 88 & 85 \\
\hline 4 & 67 & 69 & 31 \\
\hline 5 & 74 & 23 & 74 \\
\hline 6 & 81 & 20 & 42 \\
\hline 7 & 64 & 21 & 45 \\
\hline 8 & 68 & 69 & 32.44 (Mean) \\
\hline 9 & 55 & 25 & 28 \\
\hline 10 & 59 & 19 & 30 \\
\hline 11 & 76 & 20 & 75 \\
\hline 12 & 85 & 24 & 83 \\
\hline 13 & 57 & 20 & 25 \\
\hline 14 & 20 & 67 & 67 \\
\hline 15 & 22 & 53 & 28 \\
\hline 16 & 19 & 78 & 75 \\
\hline 17 & 23 & 61 & 30 \\
\hline 18 & 71 & 20 & 37 \\
\hline 19 & 83 & 24 & 80 \\
\hline 20 & 72 & 20 & 70 \\
\hline 21 & 55 & 18 & 28 \\
\hline 22 & 23 & 59 & 20 \\
\hline 23 & 25 & 63 & 36 \\
\hline 23 & 54 & 24 & 30 \\
\hline 25 & 67 & 18 & 65 \\
\hline 26 & 35 & 85 & 80 \\
\hline 27 & 25 & 69 & 63 \\
\hline 28 & 73 & 20 & 71 \\
\hline 29 & 79 & 22 & 70 \\
\hline 30 & 24 & 78 & 29 \\
\hline 31 & 25 & 79 & 41 \\
\hline
\end{tabular}

Only one patient was having bilateral sensorineural hearing loss. Of the rest 30 patients, 18 patients had right ear involvement and 13 had left side involvement (Table-1).

Twenty three patients had clinical improvement of hearing; out of these 19 patients had documented hearing improvement in pure tone audiometry closer to $30 \mathrm{~dB}$. The patient having bilateral hearing loss improved to $32 \mathrm{~dB}$ on both sides (Mean $32.44 \mathrm{~dB}$ ).

\section{DISCUSSION}

Sudden onset of sensorineural hearing loss is a rare occurrence. The exact incidence of acute sensorineural hearing loss is not known, but is estimated to be between 5 and 20 per 100,000 persons/year and varies with age. 1 This condition may be extremely distressing for the patients affected, as it has a significant impact on their ability to interact socially and they may be concerned that there is some serious underlying pathological condition. Various causes including viral. ${ }^{2-5}$ vascular. $^{6}$ or autoimmune conditions. ${ }^{7}$ have been postulated. As yet, no single causal agent has been identified and the aetiology may be multifactorial.

Many different treatment regimens have been suggested. These included the use of steroids. ${ }^{8}$ (Which may be given intratympanically). ${ }^{9,10}$ low molecular weight dextran. ${ }^{11}$ carbogen. ${ }^{12}$ hyperbaric oxygen. ${ }^{13}$ LDL apheresis. ${ }^{14}$, acyclovir. ${ }^{15}$ and even stellate ganglion block. ${ }^{16}$ There is little convincing evidence that any one treatment produces any improvement in the audiometric results. ${ }^{17}$ It is, however quite difficult for various reasons to design and conduct a study on this condition in order to show a statistically and more importantly a clinically significant difference. There are many reasons for this. First of all, the condition is relatively uncommon and recruitment of an adequate number of patients to allow a double-blind clinical trial to be conducted would be difficult. The second problem is that the disorder is most likely not the result of a single disease process. A drug may help one of the causes, but not another, thus resulting in confusing outcomes. A third difficulty is that of spontaneous recovery of hearing. A spontaneous recovery rate of approximately $60 \%$ has been quoted. ${ }^{18}$ although the ranges in the literature vary considerably (32-89\%). ${ }^{19,20}$

The prognosis is not predictable. Variables which may worsen the prognosis include increasing age of the patient, number of days before presentation, a more severe initial mean hearing loss and the presence of vertigo.21,22 $\mathrm{A}$ better prognosis may be associated with early hearing improvement. $^{23}$ and prompt administration of corticosteroids. ${ }^{8,24}$ but this is controversial. ${ }^{25}$ These factors may need to be taken into account when analysing results.

There is no general agreement regarding the definition of what constitutes acute, idiopathic sensorineural hearing loss. There is no consensus regarding the time period of evolution (In order to classify as acute) or which investigations need to be performed (In order to classify as idiopathic). One definition in the literature is "A loss of $30 \mathrm{~dB}$ at three contiguous frequencies within three days."

Panda NK et al, conducted a study regarding the efficacy of transcutaneous application of nitroglycerin along with oral steroid. There was a clinically significant improvement in hearing in $36.2 \%$ of the patients. A significant association was seen between the duration of hearing loss before seeking treatment and the recovery. Younger patients showed better improvement. The severity of hearing loss at presentation had no influence on the recovery. ${ }^{26}$ Our patients had similar rate of hearing improvement.

Dextran 40 is still available, but has recently not been prescribed due to concerns raised regarding its safety. ${ }^{26}$ especially in elderly patients. Low molecular weight dextran increases plasma viscosity and should be used with caution in patients with heart disorders or renal impairment, since pulmonary. ${ }^{27}$ or renal failure..$^{28}$ may result and deaths have been reported. ${ }^{29}$ Whilst this infusion is being administered, the patient's urine and haematocrit should be closely monitored.

A Magnetic Resonance Imaging (MRI) scan of the internal auditory meatus should be performed in all cases. ${ }^{30}$ even if their hearing loss improves. ${ }^{31,32} \mathrm{~A}$ study of 67 patients with acute sensorineural hearing loss by Nageris and Popovtzer found 24 patients had an acoustic neuroma; of these 4 (16.7\%) recovered hearing after 1 month. 33 Rarely, a vestibular 
schwannoma may present in this manner.34-37 Further investigations may help to identify factors, which contribute to the hearing loss.

The United States National Institute of Health is currently running a multi-centre randomised controlled trial. 38 to compare the efficacy of oral prednisolone and intratympanic methyl-prednisolone for the treatment of moderate-to-severe sensorineural hearing loss.

\section{CONCLUSION}

Sudden onset sensorineural hearing loss is an ENT emergency and patient need to be present early in order to get best result. Transdermal nitroglycerin patch is a supportive therapy along with intravenous pulse steroid. This regimen was found to be efficacious in the treatment of SSNHL. The single most important factor that influenced the recovery was the duration of hearing loss before seeking treatment. All patients should have an MRI scan, even if they have recovered from the hearing loss.

\section{REFERENCES}

1. Byl FM. Sudden hearing loss: eight years' experience and suggested prognostic table. Laryngoscope 1984;94(5 Pt 1):647-61.

2. Wilson WR, Veltri RW, Laird $\mathrm{N}$, et al. Viral and epidemiological studies of idiopathic sudden hearing loss. Otolaryngol Head Neck Surg 1983;91(6):653-8.

3. Takasaki T, Higashikawa M, Motoyama S, et al. Serum antibodies to human herpes virus 7, human herpes virus 6 and cytomegalovirus in patients with idiopathic facial nerve palsy or sudden deafness. J Laryngol Otol 1998;112(7):617-21.

4. Mentel R, Kaftan H, Wegner U, et al. Are enterovirus infections a co-factor in sudden hearing loss? J Med Virol 2004;72(4):625-9.

5. Schattner A, Halperin D, Wolf D, et al. Enteroviruses and sudden deafness. CMAJ 2003;168(11):1421-3.

6. Belal A. Pathology of vascular sensorineural hearing impairment. Laryngoscope 1980;90(11 Pt 1):1831-9.

7. Berrocal JR, Ramirez-Camacho R. Sudden sensorineural hearing loss: supporting the immunologic theory. Ann Otol Rhinol Laryngol 2002;111(11):989-97.

8. Wilson W, Byl FM, Laird N. The efficacy of steroids in the treatment of idiopathic sudden hearing loss. A double blind clinical study. Arch Otolaryngol 1980;106(12):7726.

9. Chandrasekhar SS. Intratympanic dexamethasone for sudden sensorineural hearing loss: clinical and laboratory evaluation. Otol Neurotol 2001;22(1):18-23.

10. Fiztgerald DC, McGuire JF. Intratympanic steroids for idiopathic sudden sensorineural hearing loss. Ann Otol Rhinol Laryngol 2007;116(4):253-6.

11. Probst R, Tschopp K, Ludin E, et al. A randomised, double blind, placebo controlled study of dextran/pentoxifylline medication in acute acoustic trauma and sudden hearing loss. Acta Otolaryngol (Stockh) 1992;112(3):435-43.

12. Fisch U. Management of sudden deafness. Otolaryngol Head Neck Surg 1983;91(1):3-8.

13. Bennett M, Kertesz T, Yeung P. Hyperbaric oxygen therapy for idiopathic sudden sensorineural hearing loss and tinnitus: a systematic review of randomised controlled trials. J Laryngol Otol 2005;119(10):791-8.
14. Suckfull M, Thiery J, Schorn K, et al. Clinical utility of LDLapheresis in the treatment of sudden hearing loss: a prospective, randomised study. Acta Otolaryngol (Stockh) 1999;119(7):763-6.

15. Uri N, Doweck I, Cohen-Kerem R, et al. Acyclovir in the management of idiopathic sensorineural hearing loss. Otolaryngol Head Neck Surg 2003;128(4):544-9.

16. Haug O, Draper WL, Haug SA. Stellate ganglion blocks for idiopathic sensorineural hearing loss. Arch Otolaryngol 1976;102(1):5-8.

17. Finger RP, Gostian AO. Idiopathic sudden hearing loss: contradictory clinical evidence, placebo effects and high spontaneous recovery rate-where do we stand in assessing treatment outcomes? Acta Otolaryngol 2006;126(11):1124-7.

18. Leong HK, Loh KK. Prognostic factors in idiopathic sudden hearing loss. Ann Acad Med 1991;20(5):624-7.

19. Moskowitz D, Lee KJ, Smith HW. Steroid use in idiopathic sudden sensorineural hearing loss. Laryngoscope 1984;94(5 Pt 1):664-6.

20. Harada H, Kato T. Prognosis for sudden sensorineural hearing loss: a retrospective study using logistical regression analysis. Int Tinnitus J 2005;11(2):115-8.

21. Kitajiri S, Tabuchi K, Hiraumi H, et al. Is corticosteroid therapy effective for sudden onset sensorineural hearing loss at lower frequencies? Arch Otolaryngol Head Neck Surg 2002;128(4):365-7.

22. Ito $\mathrm{S}$, Fuse $\mathrm{T}$, Yokota $\mathrm{M}$, et al. Prognosis is predicted by early hearing improvement in patients with idiopathic sudden sensorineural hearing loss. Clin Otolaryngol 2002;27(6):501-4.

23. Jeyakumar A, Francis D, Doerr T. Treatment of idiopathic sudden sensorineural hearing loss. Acta Otolaryngol 2006;126(7):708-13.

24. Huy PT, Sauvaget E. Idiopathic sensorineural hearing loss is not an otologic emergency. Otol Neurotol 2005;26(5):896-902.

25. Kuo ST, Hsu WC, Young YH. Dextran-induced pulmonary oedema in patients with sudden deafness. Otol Neurotol 2002;23(5):661-4.

26. Panda NK, Verma RK, Sarvanan K. Sudden sensory hearing loss: have we got a cure? J Head Neck Surg 2008;37(6):807-12.

27. Zaytoun GM, Schuknecht HF, Farmer HS. Fatality following the use of low molecular weight dextran in the treatment of sudden deafness. Adv Oto-Rhino-Laryngol 1983;31:240-6.

28. Clinical effectiveness guidelines, author. Acoustic neuroma (Vestibular Schwannoma) British association of otolaryngologists. Head and Neck Surgeons BAO-HNS Document 5. 2002.

29. Berg HM, Cohen NL, Hammerschlag PE, et al. Acoustic neuroma presenting as sudden hearing loss with recovery. Otolaryngol Head Neck Surg 1986;94(1):15-22.

30. Berenholz LP, Eriksen C, Hirsh FA. Recovery from repeated sudden hearing loss with corticosteroid use in the presence of an acoustic neuroma. Ann Otol Rhinol Laryngol 1992;101(10):827-31.

31. Nagaris BI, Popovtzer A. Acoustic neuroma in patients with completely resolved sudden hearing loss. Ann Otol Rhinol Laryngol 2003;112(5):395-7. 
32. Aslan A, Donato G, Balyan FR, et al. Clinical observations on coexistence of sudden hearing loss and vestibular schwannoma. Otolaryngol Head Neck Surg 1997;117(6):580-2.

33. Yanagihara N, Asai M. Sudden hearing loss induced by acoustic neuroma: significance of small tumours. Laryngoscope 1993;103:308-11.
34. Saunders JE, Luxford WM, Kay Devgan K, et al. Sudden hearing loss in acoustic neuroma patients. Otolaryngol Head Neck Surg 1995;113(1):23-31.

35. Inoue Y, Kanzaki J, Ogawa K. Vestibular schwannoma presenting as sudden deafness. JLO 2000;114(8):589-92.

36. Hultcrantz E, Nosrati-Zarenoe R, Arlinger S. A national database could solve the issue of sudden sensorineural hearing loss. Lakartidningen 2003;100(39):3055-9. 